\title{
Antenna Analytical Representation by a Two-Port Network
}

\author{
Peter L. Tokarsky $\mathbb{D}^{1,2}$ \\ ${ }^{1}$ Department of Radio Astronomy Equipment and Observation Methods, \\ Institute of Radio Astronomy of the National Academy of Sciences of Ukraine, Kharkiv 61002, Ukraine \\ ${ }^{2}$ Department of Computer Radio Engineering and Technical Information Security Systems, \\ Kharkiv National University of Radio Electronics, Kharkiv 61166, Ukraine
}

Correspondence should be addressed to Peter L. Tokarsky; p.tokarsky@rian.kharkov.ua

Received 20 March 2020; Revised 20 May 2020; Accepted 11 June 2020; Published 24 July 2020

Academic Editor: Ping Li

Copyright (C) 2020 Peter L. Tokarsky. This is an open access article distributed under the Creative Commons Attribution License, which permits unrestricted use, distribution, and reproduction in any medium, provided the original work is properly cited.

System analysis is a powerful tool for researching modern wireless systems. This includes breaking such systems into parts that make them up and studying how these parts work together. All these parts can be represented as "black boxes" in the form of two-port or multiport networks with the common system of parameters. Antenna is an integral part of any wireless system, so it should be also represented as a two-port network. In this paper, an analytical model of an arbitrary single antenna in the form of a two-port network, whose electrical and noise parameters are described in terms of scattering matrices, is obtained. The initial data for creating the model are the antenna fundamental parameters, viz., the input reflection coefficient and the radiation efficiency. Applications of this model for antenna analysis operating in the transmitting, receiving, and scattering modes are demonstrated. A numerical example using the antenna scattering matrix for computer simulation of a wireless connection is given.

\section{Introduction}

The IEEE standard [1] defines an antenna as "a terminal devise of a transmitting or receiving system which is designed to radiate or receive electromagnetic waves"; thereunder the antenna operating in the transmission mode is usually represented as the load impedance and in the receiving mode as the Thevenin or Norton equivalent generator $[2,3]$. At the same time, the antenna can also be viewed as a transducer that converts waves traveling in a transmission line to waves propagating in free space, and vice versa $[4,5]$. Such mutual conversions are often accompanied by energy losses, which not only reduce the conversion efficiency, but also generate thermal noise that can affect the receiving system sensitivity. Therefore, often there is a need to represent an antenna in the form of a two-port network, whose one terminal is connected to the transmission line running to a generator or receiver, and the second one is connected to a transmission line simulating the energy exchange channel with free space.

One of the first attempts to represent an antenna in the form of an electromagnetic wave converter was made in [6], where the field in free space was presented as an infinite sum of spherical harmonics, which made this antenna model inconvenient for use. Further attempts of creating an antenna two-port network were made repeatedly [7-17]. The authors in [7-13] used a theoretical approach; however, unfortunately, they did not obtain a complete analytical description of the antenna two-port network. In [14-16], the authors developed a technique for the experimental determination of S-parameters of the antenna two-port network, which is based on the Wheeler cap method. However, the application of the method is limited to the microwave range, when the manufacture of the cap does not cause difficulties. It is clear for the antennas of HF and of lower frequency ranges that this method is practically not applicable for obvious reasons. The analytical form of the two-port network S-matrix was derived from [17] for the dipole antenna, but the specific topic of the paper did not allow the authors to generalize those results to arbitrary antennas.

In this paper, an extended theory of a two-port network associated with an arbitrary single-input antenna is developed. The electric and noise parameters of this network (Figure 1) are described in terms of wave matrices by the following equations [18]: 


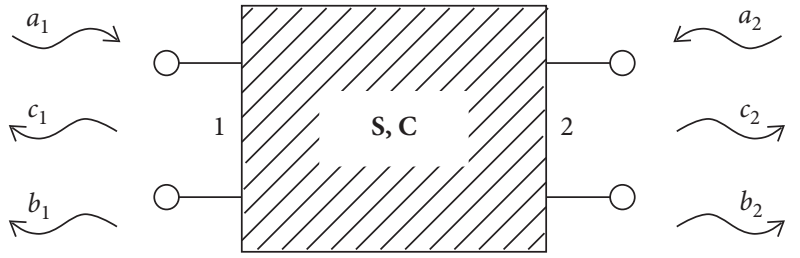

Figure 1: A two-port network associated with the antenna.

$$
\begin{aligned}
& \dot{b}_{1}=S_{11} \dot{a}_{1}+S_{12} \dot{a}_{2}+\dot{c}_{1}, \\
& \dot{b}_{2}=S_{21} \dot{a}_{1}+S_{22} \dot{a}_{2}+\dot{c}_{2},
\end{aligned}
$$

where $S_{m n}(m, n=1,2)$ are scattering matrix elements of the two-port network; $\dot{a}_{1}, \dot{a}_{2}$ and $\dot{b}_{1}, \dot{b}_{2}$ are normalized complex amplitudes of the incident and reflected harmonic waves at the input 1 and output 2 ports, as is shown in Figure $1 ; \dot{c}_{1}$ and $\dot{c}_{2}$ are complex amplitudes of the noise waves outgoing from the two-port network. The sources of these waves are inside the antenna; their mean values are $\overline{\dot{c}_{1,2}}=0$, and spectral densities are characterized by the covariance matrix $\mathbf{C}$ of noise waves:

$$
\mathbf{C}=\left(\begin{array}{cc}
\overline{\dot{c}_{1} \dot{c}_{1}^{*}} & \overline{\dot{c}_{1} \dot{c}_{2}^{*}} \\
\overline{\dot{c}_{2} \dot{c}_{1}^{*}} & \overline{\dot{c}_{2} \dot{c}_{2}^{*}}
\end{array}\right)=\left(\begin{array}{ll}
C_{11} & C_{12} \\
C_{21} & C_{22}
\end{array}\right) \text {. }
$$

In Section 2, the scattering matrix for a dipole-type antenna is found. In Section 3, the scattering matrix for an arbitrary single antenna is derived. In Section 4, the noise C-matrix for both dipole and arbitrary antenna is determined. In Section 5, application of the obtained matrices for determining antenna parameters operating in transmission, reception, and scattering modes is illustrated. In Section 6, a numerical example using the antenna scattering matrix for computer simulation of a wireless connection is given.

\section{Dipole S-Matrix}

Consider a dipole antenna, assuming that we know its normalized radiation pattern $F(\theta, \phi)$, maximum directivity $D_{m}$, radiation efficiency $\eta$, and impedance $Z=R+j X=R_{r}+R_{l}+j X$, where $R_{r}=\eta R$ is the radiation resistance, $R_{l}=(1-\eta) R$ is the loss resistance, and $X$ is reactance. Figure 2(a) shows the well-known Thevenin equivalent circuit of a dipole operating in the transmission mode [2]. The radiation resistance $R_{r}$ shown here is a principal element of the dipole equivalent circuit, since without it the dipole ceases to radiate. The other two circuit elements, $R_{l}$ and $X$ in Figure 2(a), are the parasitic elements, which do not take part in the radiation process. The first of them reduces the antenna efficiency, and the second one limits the possibility of matching the dipole with the generator, so they as a rule are trying to get rid of them, or at least to minimize them as much as possible.

We present the circuit shown in Figure 2(a) as a cascaded connection of two two-port networks (Figure 2(b)). The first of them is a series impedance $Z_{p}$ inserted in a transmission line of characteristic impedance $Z_{c}$. Its scattering matrix is [19]

$$
\mathbf{S}_{\mathrm{I}}=\frac{1}{Z_{p}+2 Z_{c}}\left(\begin{array}{cc}
Z_{p} & 2 Z_{c} \\
2 Z_{c} & Z_{p}
\end{array}\right)
$$

The second two-port network II converts the waves propagating along the transmission line into waves propagating in free space, i.e., it corresponds to an ideal lossless antenna. Its input impedance is a resistor $R_{r}$ that is virtual, because the power in it does not convert into heat, but is radiated into free space. We find the scattering matrix $\mathbf{S}_{\mathrm{II}}$ of the two-port network II, assuming that its input terminals are connected to a transmission line of the characteristic impedance $Z_{c}$, and the output terminals are connected to a virtual transmission line that simulates free space. The characteristic impedance $Z_{w}$ of a virtual line remains to be determined. The element $S_{\text {II } 11}$ of the desired matrix is the input reflection coefficient $\Gamma_{r}$ :

$$
S_{\mathrm{II} 11}=\Gamma_{r}=\frac{R_{r}-Z_{c}}{R_{r}+Z_{c}} .
$$

Other S-matrix elements can be obtained using its symmetry and unitarity properties [19]:

$$
\left|S_{\mathrm{II} 21}\right|^{2}=1-\left|S_{\mathrm{II} 11}\right|^{2}=1-\left|\Gamma_{r}\right|^{2}
$$

from which follows

$$
\begin{aligned}
& S_{\mathrm{II} 12}=S_{\mathrm{II} 21}=\sqrt{1-\left|\Gamma_{r}\right|^{2}} e^{-j \varphi}=\frac{2 \sqrt{R_{r} Z_{c}}}{R_{r}+Z_{c}} e^{-j \varphi}, \\
& S_{\mathrm{II} 22}=S_{\mathrm{II} 11}^{*} \frac{S_{\mathrm{II} 12}}{S_{\mathrm{II} 21}^{*}}=-\Gamma_{r}^{*} e^{-j 2 \varphi}=-\frac{R_{r}-Z_{c}}{R_{r}+Z_{c}} e^{-j 2 \varphi},
\end{aligned}
$$

where $\varphi$ is an arbitrary phase.

As a result, the obtained S-matrix of the lossless antenna has the form

$$
\begin{aligned}
\mathbf{S}_{\mathrm{II}} & =\left(\begin{array}{cc}
\Gamma_{r} & \sqrt{1-\left|\Gamma_{r}\right|^{2}} e^{j \varphi} \\
\sqrt{1-\left|\Gamma_{r}\right|^{2}} e^{-j \varphi} & -\Gamma_{r}^{*} e^{-j 2 \varphi}
\end{array}\right), \\
\operatorname{or} \mathbf{S}_{\mathrm{II}} & =\frac{1}{R_{r}+Z_{c}}\left(\begin{array}{cc}
R_{r}-Z_{c} & 2 \sqrt{R_{r} Z_{c}} e^{-j \varphi} \\
2 \sqrt{R_{r} Z_{c}} e^{-j \varphi} & \left(Z_{c}-R_{r}\right) e^{-j 2 \varphi}
\end{array}\right) .
\end{aligned}
$$

The sought dipole S-matrix resulting from cascading of the two two-port networks (Figure 2(b)) can be derived using [19] and expressed as follows:

$$
\mathbf{S}=\frac{1}{Z+Z_{c}}\left(\begin{array}{cc}
Z-Z_{c} & 2 \sqrt{Z_{c} R_{r}} e^{-j \varphi} \\
2 \sqrt{Z_{c} R_{r}} e^{-j \varphi} & \left(Z+Z_{c}-2 R_{r}\right) e^{-j 2 \varphi}
\end{array}\right) .
$$

As follows from [19], the scattering matrix $\mathbf{S}$ in (9) describes also a two-port network in the form of the serial impedance $Z_{p}=R_{l}+j X$ inserted between two transmission lines of the different characteristic impedances $Z_{c}$ and $Z_{w}=$ $R_{r}$ (Figure 3). However, the radiation resistance of the antennas is not very suitable for this role, since they, as a rule, depend on the frequency. To get rid of this drawback, we will add an $n: 1$ transformer to the circuit (Figure 3), which 


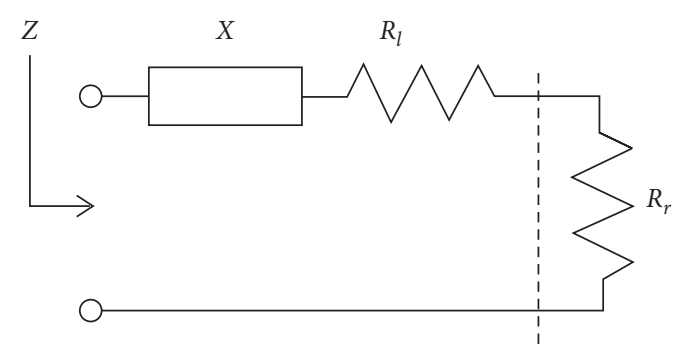

(a)

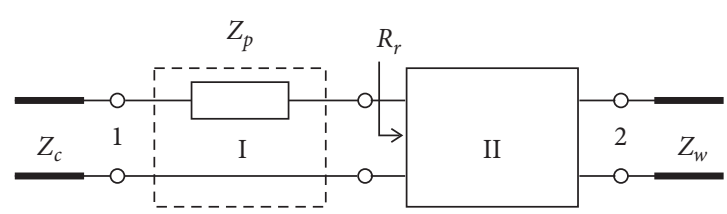

(b)

Figure 2: An equivalent circuit (a) of the dipole and its representation as a two-port network (b).

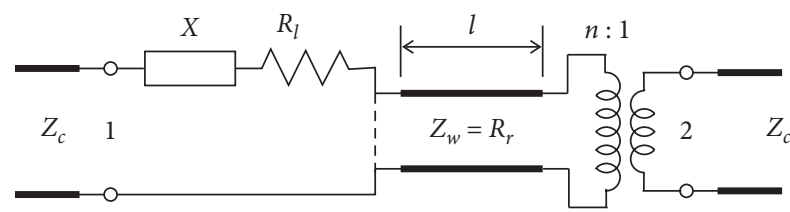

Figure 3: Equivalent circuit of a dipole in the form of a two-port network.

transforms the resistance $R_{r}$ to $Z_{c}$, and select its transformation coefficient as $n=\sqrt{R_{r} / Z_{c}}$. Note that the addition of a transformer does not change the scattering matrix $\mathbf{S}$ (9) of the entire four-terminal network since the scattering matrix of this transformer is a unity matrix. The transmission line section included into the two-port network shifts out the port reference plane on distance $l$ producing the phase delay $\varphi=2 \pi l / \lambda_{0}$ in (8), where $\lambda_{0}$ is the wavelength in free space.

Both the equivalent circuit shown in Figure 3 and expression (9) can be used to calculate the scattering matrix not only of a dipole antenna but also for any other antenna having a uniquely defined terminals voltage $V_{A}$ and current $I_{A}$. Their ensemble includes all antennas fed by the TEM transmission lines.

\section{S-Matrix of an Arbitrary Antenna}

There are the dipole impedance $Z$ and the radiation resistance $R_{r}$ in (9); however, for many antennas these parameters cannot be unambiguously determined. Among them are horns, slotted waveguide antennas, and others, fed by waveguides in which the TEM waves cannot exist. For characterizing such antennas, the input reflection coefficient $\Gamma$ and efficiency $\eta$ are used. We express the scattering matrix (9) in terms of $\Gamma$ and $\eta$ using such substitutions $\Gamma=(Z-$ $\left.Z_{c}\right) /\left(Z+Z_{c}\right)$ and $\eta=R_{r} / R$. The result is

$$
\mathbf{S}=\left(\begin{array}{cc}
\Gamma & \sqrt{\eta \chi} e^{-j(\xi+\varphi)} \\
\sqrt{\eta \chi} e^{-j(\xi+\varphi)} & \frac{1-\Gamma^{*}-\eta \chi}{1-\Gamma} e^{-j 2(\xi+\varphi)}
\end{array}\right),
$$

where $\chi=1-|\Gamma|^{2}=2 \sqrt{R Z_{c}} /\left|Z+Z_{c}\right|$ is the impedance mismatch factor between the antenna terminals and transmission line; $e^{j \xi}=\left(Z+Z_{c}\right) /\left|Z+Z_{c}\right|=\left(1-\Gamma^{*}\right) /|1-\Gamma|$ is the phase delay factor ensuring the equivalence of expressions (9) and (10).
The phase delay $\varphi$ in (9) and (10) may be arbitrary, when there are no additional conditions. If we assume $\varphi=0$, (9) and (10) are somewhat simplified, but in this case the output reference plane of the two-port network shifts into location of the impedance step discontinuity. Equation (10) can be simplified even more if we set $\varphi=-\xi$ :

$$
\begin{aligned}
\mathbf{S} & =\left(\begin{array}{cc}
\Gamma & \sqrt{\eta \chi} \\
\sqrt{\eta \chi} & \frac{1-\Gamma^{*}-\eta \chi}{1-\Gamma}
\end{array}\right) \\
& =\left(\begin{array}{cc}
\frac{Z-Z_{c}}{Z+Z_{c}} & 2 \frac{\sqrt{Z_{c} R_{r}}}{\left|Z+Z_{c}\right|} \\
2 \frac{\sqrt{Z_{c} R_{r}}}{\left|Z+Z_{c}\right|} & \frac{Z+Z_{c}-2 R_{r}}{Z^{*}+Z_{c}}
\end{array}\right) .
\end{aligned}
$$

Here, the reference planes of the input and output terminals of a two-port network coincide.

\section{Antenna Noise Covariance Matrix}

We find the covariance spectral matrix $\mathbf{C}$ of the noise waves of an antenna two-port network using the Bosma theorem [20]:

$$
\mathbf{C}=k_{B} T_{0}\left(\mathbf{E}-\mathbf{S S}^{*}\right),
$$

where $T_{0}$ is the ambient temperature and $k_{B}$ is the Boltzmann constant.

Substituting (9) and (10) into (12), we obtain the following two expressions:

$$
\begin{aligned}
\mathbf{C} & =k_{B} T_{0} \frac{4 R_{l} Z_{c}}{\left|Z+Z_{c}\right|^{2}}\left(\begin{array}{cc}
1 & -\sqrt{\frac{R_{r}}{Z_{c}}} \\
-\sqrt{\frac{R_{r}}{Z_{c}}} & \frac{R_{r}}{Z_{c}}
\end{array}\right) \\
& =k_{B} T_{0}(1-\eta) \chi\left(\begin{array}{cc}
1 & -\frac{\sqrt{\eta \chi}}{|1-\Gamma|} \\
-\frac{\sqrt{\eta \chi}}{|1-\Gamma|} & \frac{\eta \chi}{|1-\Gamma|^{2}}
\end{array}\right),
\end{aligned}
$$

the first of which is convenient for calculating the covariance noise matrix of a dipole antenna and the second one for an arbitrary antenna. 


\section{Applications of the S- and C-Matrices for Antenna Analysis}

5.1. Transmitting Mode. The antenna is excited by the $a_{1}$ wave traveling from the signal generator, and there are no other sources $\left(a_{2}=0\right)$. Then, the two-port equations reduce to

$$
\begin{aligned}
& b_{1}=S_{11} a_{1}, \\
& b_{2}=S_{21} a_{1} .
\end{aligned}
$$

From the first equation, we can determine the current $I_{A}$ at the antenna input terminals as

$$
I_{A}=\sqrt{\frac{2}{Z_{c}}}\left(a_{1}-b_{1}\right)=\sqrt{\frac{2}{Z_{c}}}\left(1-S_{11}\right) a_{1}=\sqrt{\frac{2}{R_{r}}} b_{2}=S_{21} \sqrt{\frac{2}{R_{r}}} a_{1},
$$

and then find the antenna radiation field [2] at the observation point $(r, \theta, \phi)$ :

$$
\vec{E}_{r}=\frac{Z_{0} l_{e}}{2 \lambda_{0}} I_{A} \overrightarrow{\dot{F}}(\theta, \phi) \frac{e^{-j k r}}{r}=b_{2} \sqrt{\frac{Z_{0} D_{m}}{2 \pi}} \vec{F}(\theta, \phi) \frac{e^{-j k r}}{r},
$$

where $l_{e}=\lambda_{0} \sqrt{D_{m} R_{r} / \pi Z_{0}}$ is the dipole effective length; $Z_{0}$ is the intrinsic impedance of free space.

Antenna-radiated power is obtained as

$$
P_{r}=\left|b_{2}\right|^{2}=\left|S_{21}\right|^{2}\left|a_{1}\right|^{2}=\eta \chi\left|a_{1}\right|^{2} .
$$

It follows that the square of the module $S_{21}$ is the ratio of the power $P_{r}$ radiated by the antenna into free space to the wave power $P_{g}=\left|a_{1}\right|^{2}$ arriving from the signal generator by the transmission line $\left|S_{21}\right|^{2}=P_{r} / P_{a v}$.

5.2. Receiving Mode. Here, we assume the antenna being excited only by a plane EM wave which is traveling in free space and carrying the wanted signal. The wave comes from the $\left(\theta^{i}, \phi^{i}\right)$ direction, has the electrical field strength $\dot{E}^{i}$, and its polarization is matched with antenna polarization. This plane wave generates the traveling wave $\dot{a}_{2}$ in the virtual transmission line, which at port 2 of a two-port network is determined as follows:

$$
\dot{a}_{2}=\frac{V_{o c}}{2 \sqrt{2 R_{r}}}=\frac{\lambda_{0} \dot{E}^{i}}{\sqrt{2 Z_{0}}} \sqrt{\frac{D_{m}}{4 \pi}} F\left(\theta^{i}, \phi^{i}\right)
$$

where $V_{o c}=\dot{E}^{i} l_{e} F(\theta, \phi)$ is an open-circuit voltage at the dipole terminals induced by an incident plane EM wave.

The power that carries the incident wave $\dot{a}_{2}$ is

$$
P_{a v}=\left|\dot{a}_{2}\right|^{2}=\frac{\dot{E}^{i}}{\sqrt{2 Z_{0}}} \frac{\lambda_{0}^{2} D_{m}}{4 \pi} F\left(\theta^{i}, \phi^{i}\right)=W^{i} A_{a}\left|F\left(\theta^{i}, \phi^{i}\right)\right|^{2},
$$

where $A_{\alpha}=\lambda_{0}^{2} D_{m} / 4 \pi$ is an antenna absorption area [21] related to the effective area as $A_{e}=\eta A_{\alpha}, W^{i}=\left|\dot{E}^{i}\right|^{2} / 2 Z_{0}$ is the power flux density of an incident plane wave.
It should be noted that $P_{a v}$ is the available power, which an antenna can extract from an incident plane wave.

Since other excitation sources are absent $\left(\dot{a}_{1}=0\right)$, the first of the two-port network equations (1) is reduced to the form of $b_{1}=S_{12} a_{2}$. The signal power received by the antenna is determined as

$$
P_{\text {rec }}=\left|b_{1}\right|^{2}=W^{i} A_{a} \eta \chi\left|F\left(\theta^{i}, \phi^{i}\right)\right|^{2}=W^{i} A_{e} \chi\left|F\left(\theta^{i}, \phi^{i}\right)\right|^{2} .
$$

It follows from (20) and (21) that the square of the module $S_{12}$ is the ratio of the power $P_{\text {rec }}$, transferred by the antenna into the matched transmission line, to the available power $P_{a v}$, which can be extracted from the incident wave $\left|S_{12}\right|^{2}=P_{\text {rec }} / P_{a v}$.

The intrinsic noise power $P_{N}$ sent by the antenna to the receiver can be calculated from the C-matrix (14):

$$
P_{N}=B C_{11}=B k_{B} T_{0}(1-\eta) \chi,
$$

where $B$ is the receiver bandwidth.

5.3. Scattering Mode. An incident electromagnetic wave induces currents on the antenna which produce a scattering field. The total antenna scattering field $\overrightarrow{\dot{E}}^{s}$ can be represented as a sum of the structural $\overrightarrow{\dot{E}}_{\text {st }}^{s}$ (residual) and the reradiated $\overrightarrow{\dot{E}}_{\text {re }}^{s}$ fields [22]. The S-matrix approach allows calculating only the reradiated field. We find this field for the antenna terminated with the load impedance $Z_{L}$. In this case, the second equation in (1) will have the form

$$
b_{2}=\Gamma_{2} a_{2}
$$

where

$$
\begin{aligned}
& \Gamma_{2}=S_{22}+\frac{S_{21} S_{12} \Gamma_{L}}{1-\Gamma_{L} S_{11}}, \\
& \Gamma_{L}=\frac{Z_{L}-Z_{c}}{Z_{L}+Z_{c}} .
\end{aligned}
$$

For determination of the antenna reradiated field, we can use (15), which in view of (23) takes the form

$$
\overrightarrow{\dot{E}}_{\mathrm{re}}^{s}=a_{2} \Gamma_{2} \sqrt{\frac{Z_{0} D_{m}}{2 \pi}} \overrightarrow{\dot{F}}(\theta, \phi) \frac{e^{-j k r}}{r} .
$$

The antenna absorbed power is given by

$$
P_{a b}=\left|a_{2}\right|^{2}-\left|b_{2}\right|^{2}=\left(1-\left|\Gamma_{2}\right|^{2}\right)\left|a_{2}\right|^{2} \text {. }
$$

When $\Gamma_{2}=0$, the absorbed power reaches the maximum $P_{a b}=P_{a v}$.

When $\Gamma_{L}=0$, then $\Gamma_{2}=S_{22}$ and the absorbed power is

$$
P_{a b 0}=\left(1-\left|S_{22}\right|^{2}\right)\left|a_{2}\right|^{2} \text {, }
$$

and the reradiated power is

$$
P_{r e 0}=\left|S_{22}\right|^{2}\left|a_{2}\right|^{2} \text {. }
$$

From this equation, it follows that the square of the module $S_{22}$ is the ratio of the power $P_{r e 0}$, reradiated by the 
antenna into free space, when the transmission line is terminated by a nonreflective load, to the available power $P_{a v}$, which can be extracted from the incident wave $\left|S_{22}\right|^{2}=P_{r e 0} / P_{a v}$.

Now, using (24) and (25), we find the load impedance $Z_{L 0}$ that ensures $\Gamma_{2}=0$ :

$$
Z_{L 0}=2 R_{r}-Z=Z^{*}-2 R_{l} \text {, }
$$

or the corresponding load reflection coefficient

$$
\Gamma_{L 0}=\frac{Z_{L 0}-Z_{c}}{Z_{L 0}+Z_{c}}=\frac{1-\Gamma^{*}-\eta \chi}{\Gamma\left(1-\Gamma^{*}\right)-\eta \chi} .
$$

For the lossless antenna $(\eta=1)$, the condition $Z_{L}=Z_{L 0}$ $\left(\Gamma_{L}=\Gamma_{L 0}\right)$ corresponds with the conjugate matching condition $Z_{L}=Z^{*}\left(\Gamma_{L}=\Gamma^{*}\right)$, when the total absorbed power is delivered to load. For a lossy antenna, the impedance $Z_{L 0}$ in the form of a passive load can be realized only when $\eta \geq 0.5$ $\left(R_{r} \geq R_{l}\right)$, since otherwise $R_{L}=\operatorname{Re}\left(Z_{L 0}\right)$ should be negative.

And finally, the structural scattering field $\overrightarrow{\dot{E}}_{\text {st }}^{s}$ is the total scattering field of the antenna loaded with impedance $Z_{L}=Z_{L 0}$, since there is no $\overrightarrow{\dot{E}}_{\text {re }}^{s}$ field (27) when $\Gamma_{2}=0$.

\section{Numerical Example}

Consider the signal transmission between two active antennas, which are components of a wireless communication connection (Figure 4 ) in the $2.4 \mathrm{GHz}$ range.

Both active antennas, transmitting (ATA) and receiving (ARA), consist of an amplifier and a microstrip patch antenna. The antennas terminals are connected to transmission lines with an impedance of $Z_{c}=50 \mathrm{ohms}$, which lead to the transmitter and receiver. The distance between the antennas $r$ satisfies the far zone condition. Both ATA and ARA use identical rectangular patch antennas (Figure 5) with the following dimensions: length $L=33 \mathrm{~mm}$, width $W=35 \mathrm{~mm}$, feed point offset $\Delta=4 \mathrm{~mm}$, substrate thickness $t=2.5 \mathrm{~mm}$, its relative permittivity $\varepsilon_{r}=3.2$, and $\tan \delta=0.001$.

The geometry of the patch antenna was first calculated using the TL model $[23,24]$ and then refined by tuning in the NI AWR Design Environment [25]. Figure 6 shows the frequency dependence of the antenna impedance.

The antenna gain was determined by integrating its radiation pattern obtained as a result of the simulation; its maximum is $9.1 \mathrm{~dB}$ at $2.4 \mathrm{GHz}$; with a deviation of $\pm 0.4 \mathrm{GHz}$, it dropped to $6.8 \mathrm{~dB}$. The radiation efficiency was determined by the formula [23]; it decreases linearly from $-0.45 \mathrm{~dB}$ at $2 \mathrm{GHz}$ to $-0.67 \mathrm{~dB}$ at $3 \mathrm{GHz}$, and its value at $2.4 \mathrm{GHz}$ is $-0.54 \mathrm{~dB}$. These data made it possible to calculate the patch antenna scattering matrix (11); the magnitudes of its elements are shown in Figure 7 as a function of frequency.

Figure 8 shows a detailed schematic model of the ATA created in the Analog AWR Design Environment, where the MPA is represented by the "Patch" subcircuit, the S-parameters of which were set by the file in Touchstone format. The other circuit elements relate to the power amplifier, among which there is a transistor MGF0904a, as well as

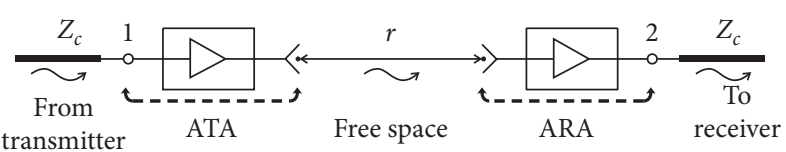

Figure 4: A wireless connection using two active antennas.

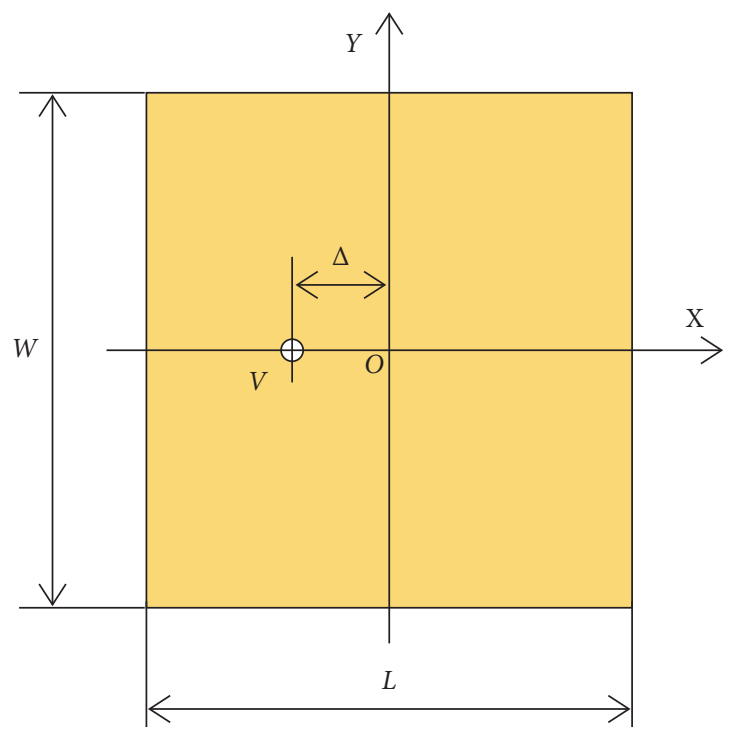

Figure 5: Patch antenna fed by via-port (V).

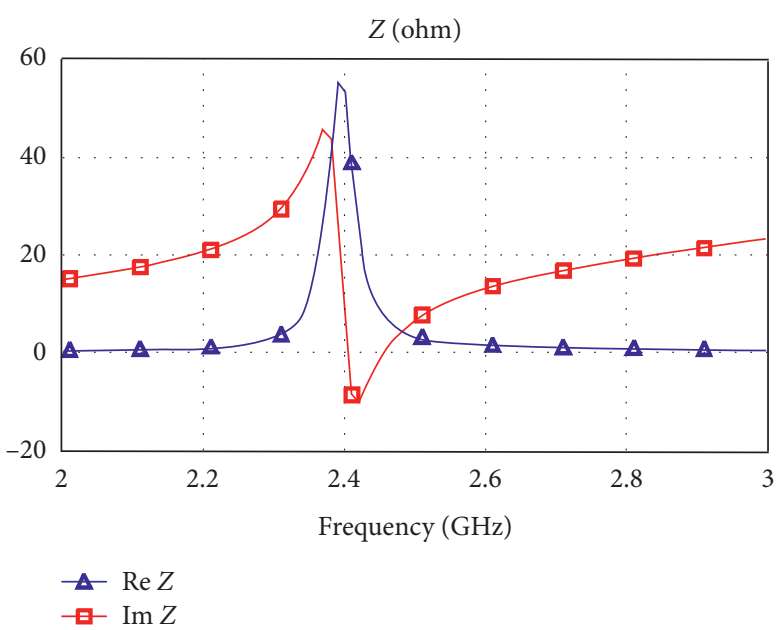

Figure 6: Patch antenna impedance $Z$ vs. frequency.

input and output matching circuits on the transmission line segments and open-circuited stubs.

A similar circuit model of the ARA is shown in Figure 9. It consists of the same patch antenna and a low-noise amplifier, consisting of a BFP740 transistor and an input matching circuit.

The matching circuits were tuned in such a way as to obtain the greatest gain of each of the two-port networks; their optimization was not carried out since we did not set ourselves such a task. Figure 10 shows the frequency dependencies of the transducer power gains $G=20 \log \left|S_{21}\right|$ [26] of 


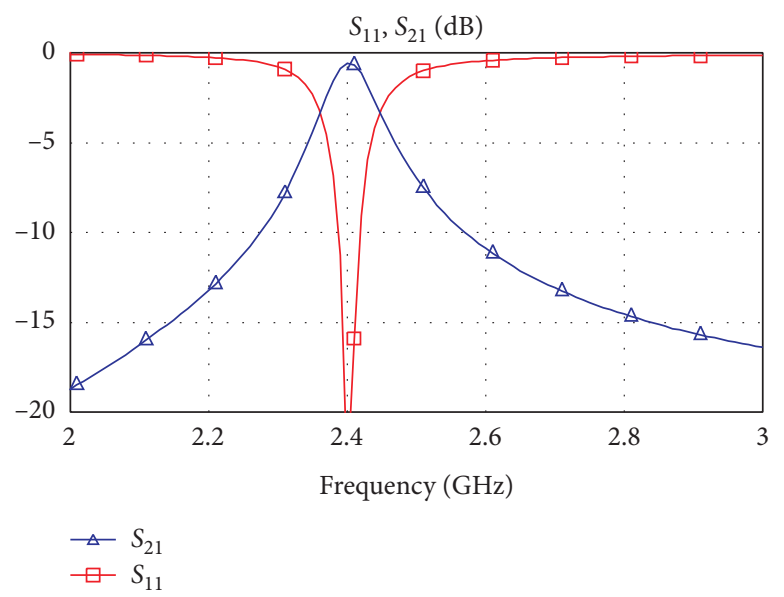

FIGURE 7: Magnitudes of elements of the patch antenna scattering matrix.

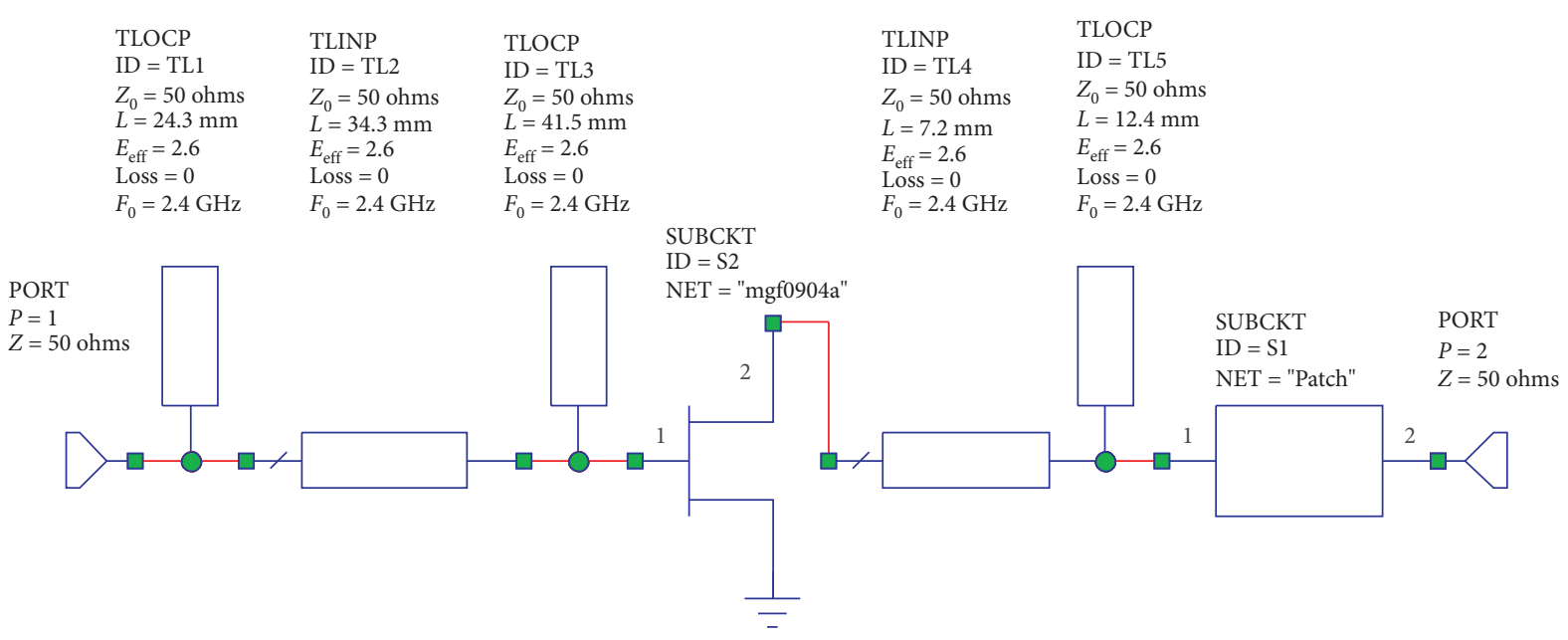

FIgURE 8: A two-port network model of the active transmitting antenna ATA.

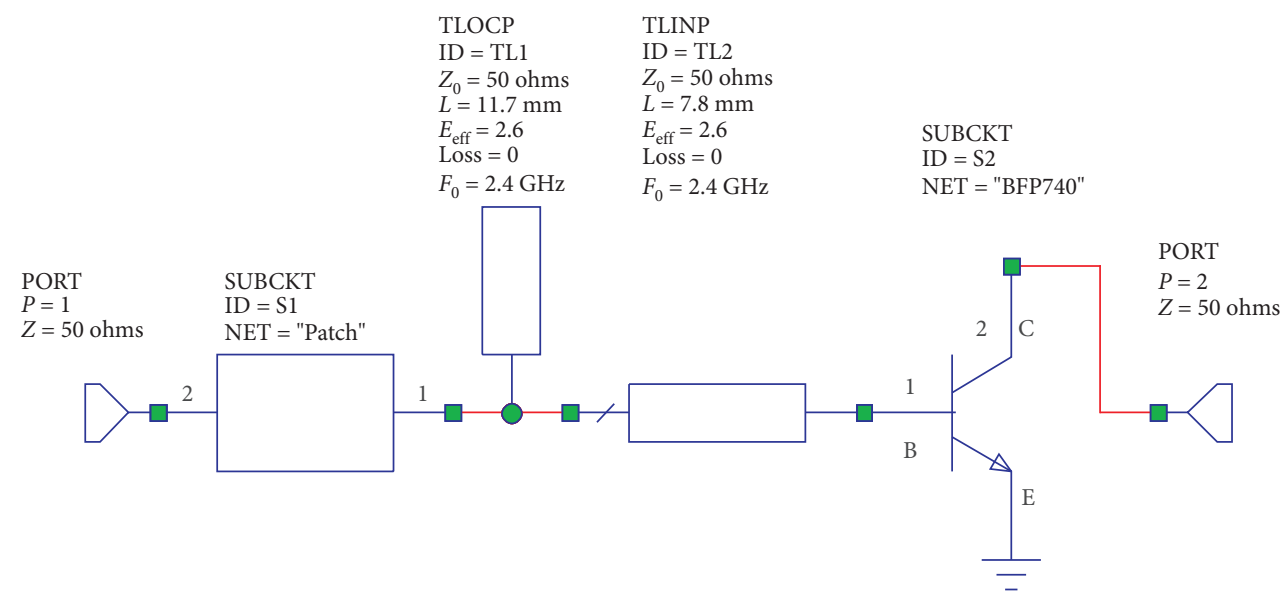

FIGURE 9: A two-port network model of the active receiving antenna ARA. 


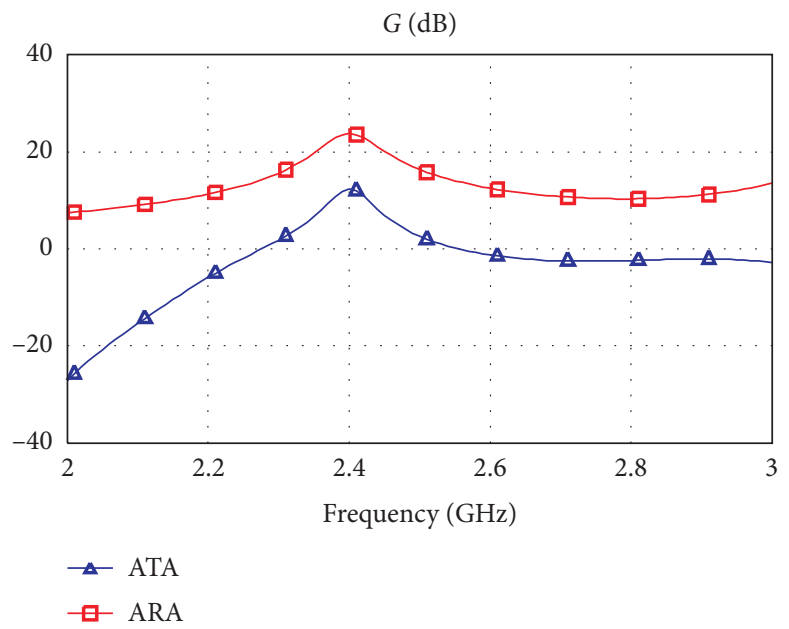

Figure 10: Transducer power gains of the active antennas two-port networks models as functions of frequency.

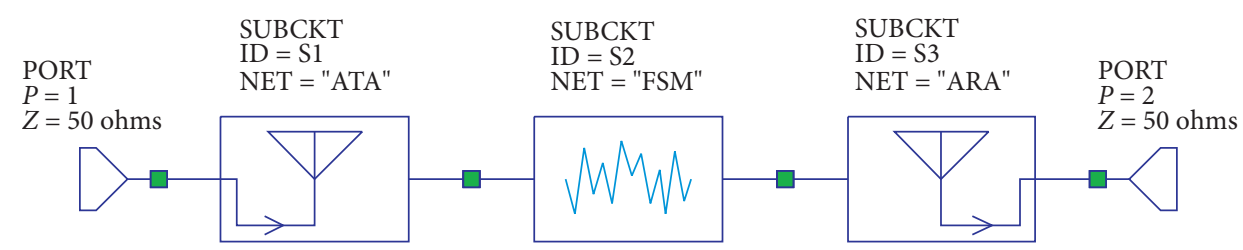

FIGURE 11: A two-port network associated with the wireless connection.

these two-port networks obtained by simulating them in the Analog AWR Design Environment. Both curves in Figure 10 have maxima at $2.4 \mathrm{GHz}$, which differ markedly from each other, $23.8 \mathrm{~dB}$ for ARA and $12.3 \mathrm{~dB}$ for ATA, since different amplifiers are used in these active antennas.

Now, we can determine the parameters of the wireless connection (WLC) shown in Figure 4. We represent it as three cascaded two-port networks (Figure 11), two of which are associated with active antennas, the parameters of which are known, and the third, FSM (Free Space Module), with a signal propagation between them. Find the S-parameters of the two-port network FSM.

Find the S-parameters of the two-port network FSM. Believing that there are no reflecting objects on the wave propagation path between the antennas, we can assume $S_{11 \mathrm{FSM}}=S_{22 \mathrm{FSM}}=0$. Parameter $S_{21 \mathrm{FSM}}$ is not difficult to determine using (17) and (19):

$$
S_{21 \mathrm{FSM}}=-j \frac{\lambda_{0} e^{-j k r}}{4 \pi r} p \sqrt{D_{m \mathrm{ARA}} D_{m \mathrm{ATA}}} F_{\mathrm{ARA}}\left(\theta^{i}, \phi^{i}\right) F_{\mathrm{ATA}}(\theta, \phi),
$$

where $p$ is the polarization mismatch factor, indices ${ }_{\mathrm{ATA}}$ and ARA denote the quantities belonging to the corresponding antennas, $(\theta, \phi)$ is the direction from ATA to ARA, and $\left(\theta^{i}, \phi^{i}\right)$ is the direction from ARA to ATA.

If the antennas are polarization matched and the radiation pattern maximum of each is directed to the other antenna, then

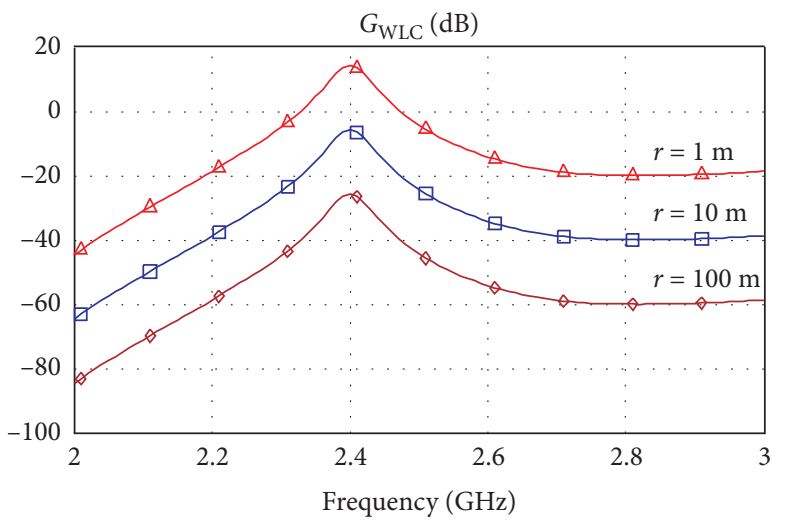

FIGURE 12: The wireless connection gain as functions of frequency.

$$
S_{21 \mathrm{FSM}}=-j \frac{\lambda_{0} e^{-j k r}}{4 \pi r} \sqrt{D_{m \mathrm{ARA}} D_{m \mathrm{ATA}}} .
$$

Since the two-port network FSM is reciprocal, then $S_{12 \text { FSM }}=S_{21 \text { FSM }}$. When antennas are identical $D_{m A R A}=D_{m A T A}=D_{m}$,

$$
S_{21 \text { FSM }}=S_{12 \text { FSM }}=-j \frac{D_{m}}{4 \pi} \frac{\lambda_{0}}{r} e^{-j k r} .
$$

Figure 12 shows the frequency dependencies of the transducer power gain of the wireless connection (34) for three distances $r$ between the antennas, $1 \mathrm{~m}, 10 \mathrm{~m}$, and 
$100 \mathrm{~m}$, obtained by simulating the circuit of Figure 11 in the NI AWR DE. The gain maximum at $r=1 \mathrm{~m}$ is $14.3 \mathrm{~dB}$, with an increase in the distance by a factor of 10; it drops by $20 \mathrm{~dB}$.

In fact, the two-port network in Figure 12 is a computer model of the Friis formula that takes into account in detail the parameters of all the elements included in the wireless connection. This data can help correct choosing of the transmitter power and receiver sensitivity when designing a wireless network.

\section{Conclusion}

The scattering matrix and the covariance noise matrix of a two-port network simulating an arbitrary single antenna are presented in an analytical form. The initial parameters for matrices calculation are the antenna input reflection coefficient and radiation efficiency. The technique of applying the scattering matrix to solve the problems of antenna analysis in transmission, reception, and scattering modes is described. A numerical example of its use for computer simulation of a wireless connection with active antennas as terminal devices is given. The simulation results allow us to correctly evaluate the frequency dependence of the wireless connection performance with a detailed accounting for all elements' parameters of the active antennas included in its structure. The proposed approach can be useful in analyzing complex wireless systems.

\section{Data Availability}

The data used to support the findings of this study are available from the corresponding author upon request.

\section{Conflicts of Interest}

The author declares that he has no conflicts of interest regarding the publication of this paper.

\section{References}

[1] IEEE standard definitions of terms for antennas," IEEE Std 145-2013.

[2] C. A. Balanis, Antenna Theory: Analysis and Design, John Wiley \& Sons Inc., Hoboken, NJ, USA, 4th edition, 2016.

[3] S. R. Best and B. C. Kaanta, "A tutorial on the receiving and scattering properties of antennas," IEEE Antennas and Propagation Magazine, vol. 51, no. 5, pp. 26-37, 2009.

[4] J. D. Kraus and R. J. Marhefka, Antennas for All Applications, McGraw Hill, New York, NY, USA, 2nd edition, 1997.

[5] W. L. Stutzman and G. A. Thiele, Antenna Theory and Design, John Wiley \& Sons Inc., Hoboken, NJ, USA, 3rd edition, 2013.

[6] C. G. Montgomery, R. H. Dicke, and E. M. Purcell, "Principles of microwave circuits," in MIT Radiation Laboratory Series, vol. 8, McGraw Hill, New York, NY, USA, 1948.

[7] R. H. Johnston and J. G. McRory, "An improved small antenna radiation-efficiency measurement method," IEEE Antennas and Propagation Magazine, vol. 40, no. 5, pp. 40-48, 1998.

[8] S. Rogers, J. Aberle, and D. Auckland, "Two-port model of an antenna for use in characterizing wireless communications systems obtained using efficiency measurements," in
Proceedings of the IEEE AP-S International Symposium, San Antonio, TX, USA, 2002.

[9] S. D. Rogers, J. T. Aberle, and D. T. Auckland, "Two-port model of an antenna for use in characterizing wireless communications systems, obtained using efficiency measurements," IEEE Antennas and Propagation Magazine, vol. 45, no. 3, pp. 115-118, 2003.

[10] J. T. Aberle, "Two-port representation of an antenna with application to non-foster matching networks," IEEE Transactions on Antennas and Propagation, vol. 56, no. 5, pp. 1218-1222, 2008.

[11] T. Prakoso, R. Ngah, and T. A. Rahman, "Representation of antenna in two-port network S-parameter," in Proceedings of the IEEE International $R F$ and Microwave Conference, pp. 293-297, Kuala Lumpur, Malaysia, December 2008.

[12] T. Prakoso, R. Ngah, Z. Ghassemlooy, and T. A. Rahman, "Antenna representation in two-port network scattering parameter," Microwave and Optical Technology Letters, vol. 53, no. 6, pp. 1404-1409, 2011.

[13] M. Haynes and M. Moghaddam, "Multipole and S-parameter antenna and propagation model," IEEE Transactions on Antennas and Propagation, vol. 59, no. 1, pp. 225-235, 2011.

[14] J. B. Coder, J. M. Ladbury, and M. Gołkowski, "A two-port model for antennas in a reverberation chamber," IEEE Transactions on Antennas and Propagation, vol. 62, no. 5, pp. 2338-2350, 2014.

[15] P. Groves, P. Conroy, L. Belostotski, and M. Okoniewski, "Measuring antenna noise parameters using a set of Wheeler caps," in Proceedings of the European Conference on Antennas and Propagation, pp. 1-4, Davos, Switzerland, April 2016.

[16] P. Groves, P. Conroy, L. Belostotski, and M. Okoniewski, "Antenna two-port electrical and noise parameters," IEEE Antennas and Wireless Propagation Letters, vol. 16, pp. 1265-1268, 2017.

[17] P. L. Tokarsky, A. A. Konovalenko, and S. N. Yerin, "Sensitivity of an active antenna array element for the low frequency radio telescope GURT," IEEE Transactions on Antennas and Propagation, vol. 65, no. 9, pp. 2540-2549, 2017.

[18] J. A. Dobrowolski, Microwave Network Design Using the Scattering Matrix, Artech House, London, UK, 2010.

[19] K. C. Gupta, R. Garg, and R. Chadha, Computer-Aided Design of Microwave Circuits, Artech House, Norwood, MA, USA, 1981.

[20] H. Bosma, On the Theory of Linear Noisy Systems, Technische Hogeschool Eindhoven, Eindhoven, Netherlands, 1967.

[21] J. B. Andersen and A. Frandsen, "Absorption efficiency of receiving antennas," IEEE Transactions on Antennas and Propagation, vol. 53, no. 9, pp. 2843-3289, 2005.

[22] R. C. Hansen, "Relationships between antennas as scatterers and as radiators," Proceedings of the IEEE, vol. 77, no. 5, pp. 659-662, 1989.

[23] T. A. Milligan, Modern Antenna Design, John Wiley \& Sons Inc., Hoboken, NJ, USA, 2nd edition, 2005.

[24] R. Bancroft, Microstrip and Printed Antenna Design, SciTech Publishing, Inc., Raleigh, NC, USA, 2nd edition, 2009.

[25] NI AWR Corp, "NI AWR design environment," 2020, https:// www.awr.com/awr-software/products/awr-designenvironment.

[26] D. M. Pozar, Microwave Engineering, John Wiley \& Sons Inc., Hoboken, NJ, USA, 4th edition, 2012. 